\title{
Checking the Realizability of BPMN 2.0 Choreographies
}

\author{
Pascal Poizat \\ University of Evry Val d'Essonne, Evry, France \\ LRI UMR 8623 CNRS, Orsay, France \\ pascal.poizat@Iri.fr
}

\author{
Gwen Salaün \\ Grenoble INP, INRIA, France \\ gwen.salaun@inria.fr
}

\begin{abstract}
Choreographies allow business and service architects to specify with a global perspective the requirements of applications built over distributed and interacting software entities. While being a standard for the abstract specification of business workflows and collaboration between services, the Business Process Modeling Notation (BPMN) has only been recently extended into BPMN 2.0 to support an interaction model of choreography, which, as opposed to interconnected interface models, is better suited to top-down development processes. An important issue with choreographies is realizability, i.e., whether peers obtained via projection from a choreography interact as prescribed in the choreography requirements. In this work, we propose a realizability checking approach for BPMN 2.0 choreographies. Our approach is formally grounded on a model transformation into the LOTOS NT process algebra and the use of equivalence checking. It is also completely tool-supported through interaction with the Eclipse BPMN 2.0 editor and the CADP process algebraic toolbox.
\end{abstract}

\section{INTRODUCTION}

Modern applications are no longer built as monolithic, stand-alone, programs. Rather, they are constructed out of the reuse and assembly of distributed and collaborating entities, e.g., business protocols, software components, or services. Software engineering processes have adapted to this, with two different viewpoints over application design and implementation. A first one is orchestration-oriented (Fig. 1). Given a set of services to be reused and the specification of the application-to-be in a centralized form, one retrieves a model of the orchestration and then implements it. Related issues are how to compose, and possibly adapt, reused services $[21,22]$ and then how to check implementation correctness [4]. This is a bottom-up approach and local perspective of service composition. However, this is not always desirable. Abstraction in the specification process promotes that the specification of a distributed application is performed

Permission to make digital or hard copies of all or part of this work for personal or classroom use is granted without fee provided that copies are not made or distributed for profit or commercial advantage and that copies bear this notice and the full citation on the first page. To copy otherwise, to republish, to post on servers or to redistribute to lists, requires prior specific permission and/or a fee.

SAC'12 March 25-29, 2012, Riva del Garda, Italy.

Copyright 200X ACM X-XXXXX-XX-X/XX/XX ...\$10.00.

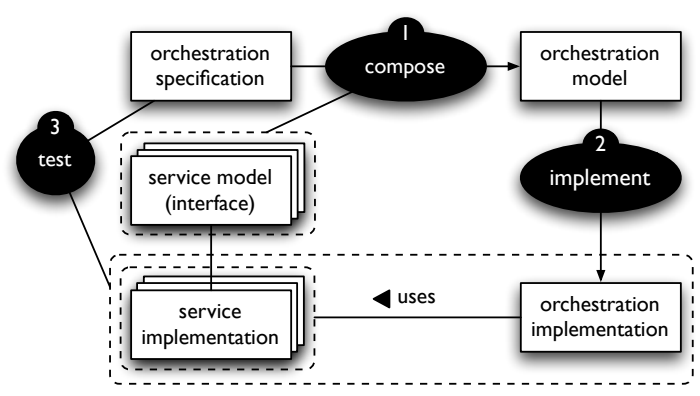

Figure 1: Orchestration-oriented development

with a global perspective on the interactions between the entities that compose it. This is the choreography-oriented viewpoint (Fig. 2).

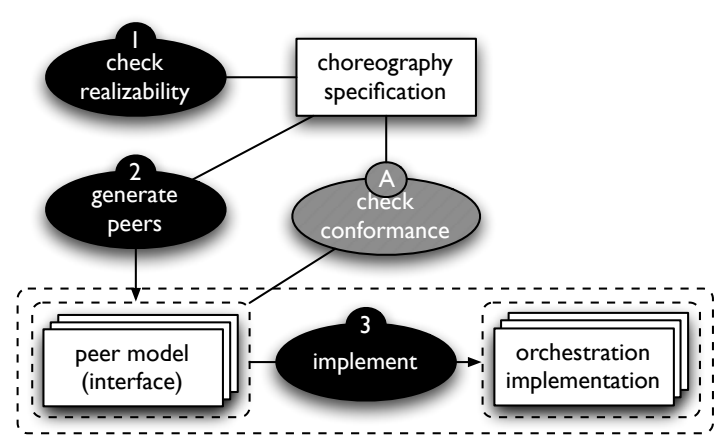

Figure 2: Choreography-oriented development

Conformance vs. realizability. In a first case, the question is whether a set of peers being reused satisfies or not the choreography specification. This is conformance checking (Fig. 2, (A)) and is again a bottom-up approach of service composition. Let $P_{i}$ denote the behaviour of some peer $i$, $\|$ denote parallel composition of the peers (including communication), and $C$ denote the expected behaviour. The conformance of a set of peers wrt. a choreography corresponds to checking equation:

$$
P_{1}\|\ldots\| P_{i}\|\ldots\| P_{n} \stackrel{?}{=} C
$$

with all $P_{i}$ and $C$ being given. Different authors have proposed techniques related to this, using different semantics for communication $(\|)$ and equivalence $(=)$, e.g., $[8,19,3]$, 
depending on the properties to be preserved between the expected behaviour and the peer composition. However, these techniques suppose that the peers are available, which is not suitable in a top-down approach. In the second case (Fig 2, $(1,2,3))$, the question is whether the choreography specification can be implemented correctly (i.e., keeping the same behaviour) by projecting it on each peer. This is realizability checking [7]. In the case that it is not, there is no need to further advance in the design process with discovery, selection, and composition of services for peers. Information should be returned back to the service architect in order to enable choreography refactoring. This corresponds to checking Equation (1) again but with each $P_{i}$ being the projection of $C$ on peer $i$.

Let us take the choreography in Figure 3, left, described with a Choreography Diagram as introduced by the BPMN 2.0 standard (see Sect. 2 for an introduction and motivation to BPMN 2.0). It specifies that $P_{1}$ and $P_{2}$ must first interact on message $M_{1}$ (with $P_{1}$ being the initiator of the interaction) and then $P_{3}$ and $P_{4}$ will interact on message $M_{2}$ (with $P_{3}$ being the initiator). It is not realizable since there is no possibility for $P_{3}$ to know that $P_{1}$ has sent (or $P_{2}$ has received) message $M_{1}$ before sending $M_{2}$. In a distributed setting, implementing $P_{1}$ (resp. $P_{3}$ ) as sending $M_{1}$ (resp. $M_{2}$ ) we could have the case where $M_{2}$ is sent before $M_{1}$ which is prevented by the choreography. Another issue is that realizability depends on the kind of communication. Let us take the choreography in Figure 3, right, and suppose that $P_{3}$ sends $M_{2}$ and that $P_{1}$ is implemented as first sending $M_{1}$ and then receiving $M_{2}$. In a synchronous setting, the choreography is realizable since $P_{3}$ will be blocked upon sending $M_{2}$ until $P_{1}$ is ready to receive it. However, in an asynchronous setting, we would have the same problem as above.
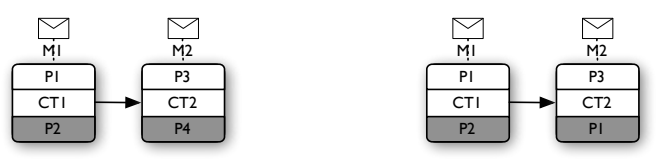

Figure 3: Examples of unrealizable choreographies

Contributions. The issue addressed in this paper is the following: given the specification of a choreography described in the BPMN 2.0 standard notation, does there exist a possible implementation of it, i.e., peer implementations obtained by projection from it, that would perform exactly as prescribed in the specification. Our first contribution is the transformation of BPMN 2.0 choreographies into a process algebra, namely LOTOS NT [10]. This paves the way for the development of formal tools dedicated to the BPMN 2.0 choreography notation, such as model-checking, testing, automatic composition, and software adaptation. Our second contribution is related to realizability. We propose techniques to check the realizability of BPMN 2.0 choreographies, thus releasing the burden on service architects when structural well-formedness constraints are required to achieve realizability. With our approach, the architect is totally free to design any desired choreography. Realizability analysis is automatically achieved as a subsequent step. Furthermore, we study realizability in both synchronous and asynchronous communication frameworks, since the kind of communication has an important impact on realizability.
This fosters the applicability of the techniques we propose. Finally, our approach is totally tool-supported, bridging design (achieved using the Eclipse BPMN 2.0 editor) and verification (achieved using the CADP verification toolbox [16]).

Organization. The rest of the paper is organized as follows. In Section 2 we advocate the use of BPMN 2.0 for the specification of choreographies and present the related notations. We also make a short introduction to the LOTOS NT process algebra. The next three sections detail our approach for BPMN 2.0 choreography analysis. Section 3 presents our formal model transformation from BPMN 2.0 choreographies to LOTOS NT processes. Section 4 explains how this encoding can be used to analyze choreographies and check their realizability, and tool support is addressed in Section 5. In Section 6 we discuss related work and compare our approach to it. Finally, Section 7 summarizes our contributions and presents perspectives of our work.

\section{BPMN 2.0 CHOREOGRAPHIES AND LOTOS NT}

\subsection{BPMN 2.0 Choreographies}

The Business Process Modeling Notation, BPMN [25], is a visual notation that well suits the needs for the specification of business processes and their interactions, including future service implementations. With reference to low-level notations or languages such as the Web Service Business Process Execution Language (WS-BPEL), BPMN promotes a high-level description that enables the service architects to focus on what services do in a composition, possibly including their conversations and interactions, without entering too much into the details of how they do it.

As classified by [12], there are two interaction models for choreography: interconnected interface models, where conversations are defined at (each) peer level and interactions are defined by roughly connecting conversations, and interaction models, where interactions between peers are the basic building blocks. The former nicely suits low-level languages such as WS-BPEL where an orchestration would be defined for each peer and communications would correspond to connections between peer models. However, from a designer perspective, and following the separation of concerns principle, interaction models better suit the needs of choreography specification due to their global perspective.

A drawback of BPMN 1.x was that it supported choreography specifications through an interconnected interface model only (Collaboration Diagrams). In contrast, the Web Service Choreography Description Language (WS-CDL) enables a real interaction model for choreography descriptions, i.e., the basic building blocks of choreographies are peer interactions. However, WS-CDL is more an implementation language than a specification notation. The lack of a standard abstract notation supporting an interaction model for choreography has been remedied with the introduction of BPMN 2.0. In addition to Collaboration Diagrams, BPMN 2.0 introduces Choreography Diagrams to support conversations with choreography tasks as a first class entity.

The basic building block of BPMN 2.0 (BPMN in the rest of this paper) Choreography Diagrams is a one-way or two-way interaction between peers. This is modelled using a choreography task (Fig. 4). Here we have two peers interacting, $A$ and $B$, represented by participant bands. $A$ is 


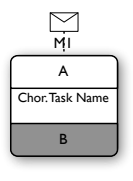

(a)
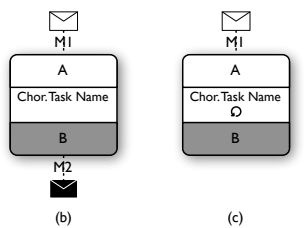

(c)

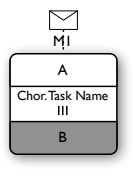

(d)

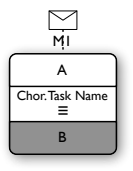

(e)
Figure 4: BPMN 2.0 notation - choreography tasks

the initiating peer, i.e., the one that decides the interaction takes place, hence it is represented by a white band as opposed to a gray filled band for B. Together with the choreography tasks, come message flows relating the interaction with an initiating message (represented by a white envelope) and, possibly, a return message (represented by a black envelope). This yields one-way interactions (Fig. 4, (a,c,d,e)) or two-way interactions (Fig. 4, (b)).

A choreography task may have an internal marker to denote whether, and how, the related interaction (one or two message exchanges) is repeated. In a standard loop (Fig. 4, (c)), the interaction is performed several times depending on a Boolean condition. In multi-instance parallel loops, the interactions are performed by several instances of the choreography task. This can be done in parallel (Fig. 4, (d)) or in sequence (Fig. 4, (e)). If the exchange is not repeated, no marker is used (Fig. 4, (a,b)).

BPMN enables one to describe control flows using sequence flows for performing two tasks in sequence or gateways for more complex behaviours. In our work we take into account the main gateways found in BPMN (Fig. 5) that is: exclusive gateways (decision, alternative paths), inclusive gateways (inclusive decision, alternative but also parallel paths), parallel gateways (creation and merging of parallel flows), and event-based gateways (choice based on events, i.e., message reception or timeout, like the WS-BPEL pick construct). We require that gateways are either diverging (multiple outgoing sequence flows and at most one incoming sequence flow) or converging (multiple incoming sequence flows and at most one outgoing sequence flow). Diagrams that would not cope to this requirement can be transformed by adding new gateways [25], e.g., a gateway being both converging and diverging can be transformed as the sequence of a converging one and a diverging one.

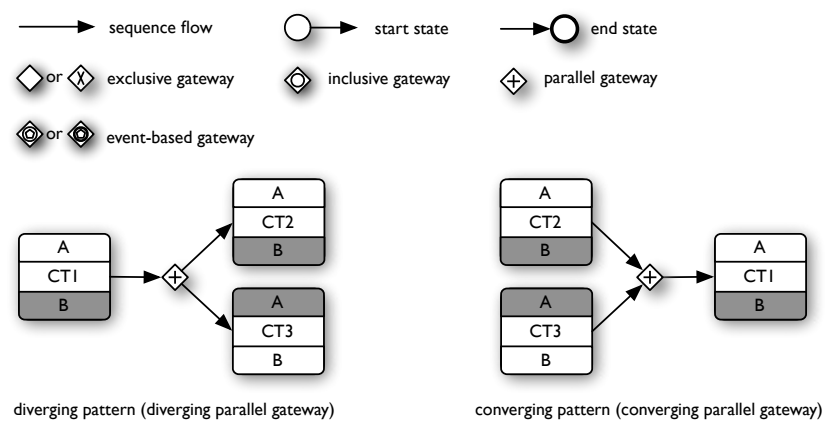

Figure 5: BPMN 2.0 notation - control flow and gateways

Running example. In this paper, we will use an e-booking system as running example. This case study involves four peers: a booking system (bs), a database (db), an on-line bank service (bk), and a client (cl). Figure 6 gives a BPMN choreography for the system under construction. In this specification, we can see that first the client interacts with the booking system (connect), submits a request to the booking system (request) and receives a response (reply). Then, the client can either choose to quit (abort), submit another request (request), or make a booking (book). In this last case, the client also pays the bank (pay), and the booking system stores some information in the database to keep track of this completed transaction (store).

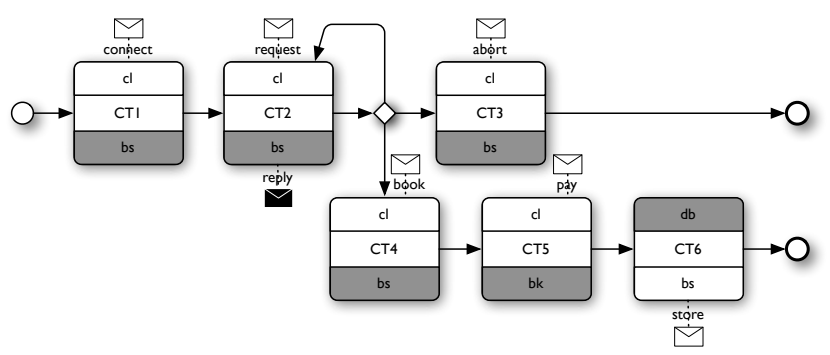

Figure 6: Running example - Booking System

\subsection{LOTOS NT}

LOTOS NT [10] is an improved version of the LOTOS ISO standard [17] that combines the best features of imperative programming languages and value-passing process algebras. LOTOS NT also supports the description of complex data types written using a functional specification language. LOTOS NT has a user-friendly syntax and formal operational semantics defined in terms of Labeled Transition Systems (LTSs).

LOTOS NT processes are built from actions, sequential compositions (;), conditions (if), assignments (:=), looping behaviours (loop), choices (select), and parallel compositions (par). Communication is carried out by rendezvous on a set of synchronization actions (multiway synchronization points) with bidirectional transmission of multiple values. Synchronizations may also contain optional guards (where) expressing Boolean conditions on received values. Processes are parameterized by sets of actions (alphabets) and input/output data variables. In the rest of this paper, we will show examples of LOTOS NT generated from BPMN choreographies for realizability checking purposes.

LOTOS NT specifications can be analysed using the CADP verification toolbox [16]. In particular, LOTOS NT is supported by the Int.open tool of CADP, which enables the on-the-fly exploration and verification of the LTSs corresponding to LOTOS NT specifications.

\section{ENCODING INTO LOTOS NT}

In this section, we present the encoding of BPMN choreographies into LOTOS NT. We chose LOTOS NT, instead of, e.g., Petri nets encodings or a direct translation into LTSs, for several reasons. First, LOTOS NT provides expressive operators for translating BPMN constructs, and a translation between two high-level languages is much simpler because both languages share similar operators, such as sequence, choice, interleaving, etc. Second, LOTOS NT is supported by state-of-the-art verification tools (CADP) that 
can be used in order to compile the LOTOS NT specification into an LTS, enumerating all the possible behaviours. The LOTOS NT operators together with the CADP tools enable us, for instance, to generate peers (Fig. 2, (2)) in a declarative way, thus avoiding the implementation of lowlevel $a d$-hoc algorithms operating on LTS models.

We encode BPMN choreographies in LOTOS NT following the state machine pattern. Each BPMN construct is encoded as a LOTOS NT process that symbolizes a state in that state machine. This process translates the behaviour of the BPMN construct plus a call to the process encoding the successor state (possibly several process calls if there are several successors, e.g., in the case of a diverging gateway). The initial choreography element identified by a start state in BPMN (e.g., CT1 in Fig. 6) is translated as a LOTOS NT process called MAIN.

We show below the LOTOS NT process encoding the second activity of our running example. This process is named CT2 and makes explicit the alphabet between squared brackets, that is, all the different messages exchanged in the choreography. These messages can be typed (with the types of their parameters), but this is optional and we use the keyword any if no type is specified. Each message is prefixed with the sender and receiver identifiers. The body of this process consists of two communications between the client and the booking system on messages request and reply. Then the LOTOS NT process (EXCL_1) encoding the next activity is called:

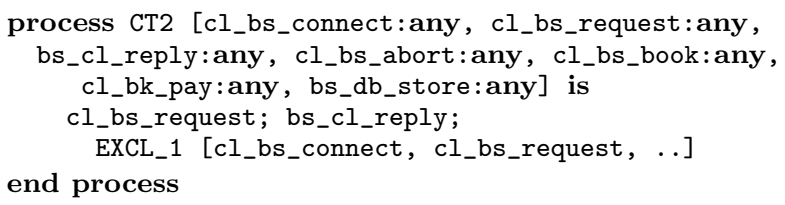

A marker may be specified in a choreography task to indicate how the messages are repeated (see Fig. 4). The standard loop is translated using the LOTOS NT loop construct, which results in a self-loop in the corresponding LTS. The sequential multi-instance is translated using the LOTOS NT sequential composition (;). The parallel multiinstance is encoded using the LOTOS NT parallel composition (par) without synchronization, meaning that all branches involved in the composition are interleaved.

Diverging and converging gateways are the most complicated part of the translation. Here, we assume that gateways are well-balanced, i.e., that for each gateway with $n$ diverging branches we have a corresponding gateway with $n$ converging branches. First of all, exclusive and inclusive gateways are translated using the LOTOS NT choice (select), whereas parallel gateways are translated using the LOTOS NT parallel operator (par). Let us show the part of our running example translating the exclusive gateway made by the client after each reply sent by the booking system. There are three possible choices, the first one corresponds to the decision of making a booking, the second choice corresponds to an abort required by the client, and the third one occurs when the client submits another request to the booking system. In each case, the process encoding the corresponding behaviour is called (CT2, СT3, and CT4 are process identifiers):

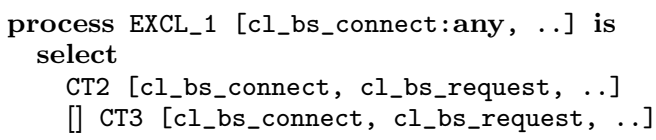

\author{
[] CT4 [cl_bs_connect, cl_bs_request, ...] \\ end select \\ end process
}

We recall that diverging inclusive gateways may fire one or more branches. Consequently, this operator is translated as a choice (select) between all possible combinations of the subsequent activities involved in the gateway. Suppose for instance that we have an inclusive gateway between two communications M1 and M2 involving three peers A, B, and C (Fig. 7, left).
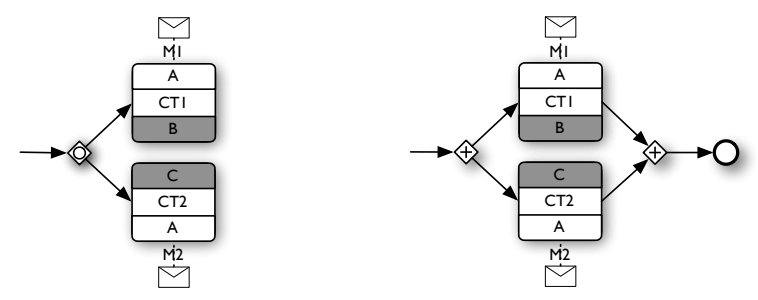

Figure 7: Examples of diverging inclusive gateway (left), diverging and converging parallel gateways (right)

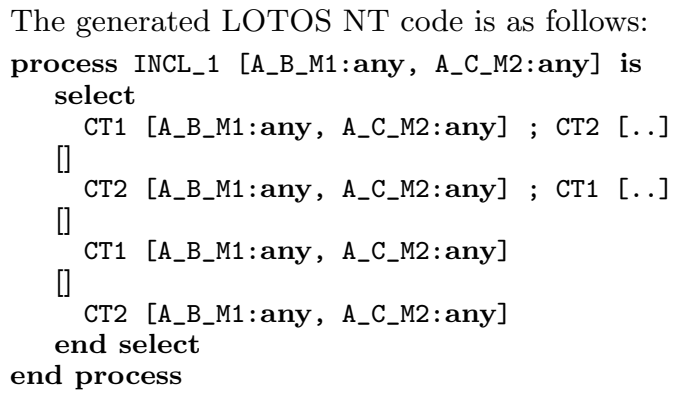

The encoding of the choreography as a state machine simplifies the translation of looping behaviours, e.g., in our example submitting as many requests as the client decides. The drawback is that some structuring information gets lost, in particular in order to translate converging gateways; one no longer knows which branches are executed in parallel and need to be merged. Therefore, to translate correctly converging/diverging gateways, we compute and generate further information. This only concerns parallel and inclusive gateways where several branches can be executed at the same time, and for which we may need to generate a proper merge (a single branch is executed in an exclusive gateway). Let us illustrate this with a simple example where we have successively a diverging parallel gateway followed by two interactions M1 and M2, and a converging parallel gateway (Fig. 7, right). In such a case, we need a synchronization point to merge the different branches involved in this gateway. To do so, when we translate the diverging gateway, we traverse the specification forward to detect a possible converging gateway. If there is a converging gateway, we generate an additional branch in the LOTOS NT parallel composition with a synchronization on an arbitrary action sync_1. After this action, we translate the rest of the choreography (process PAR_2 below). Once processes CT1 and CT2 have completed their behaviour, they can synchronize on sync_1 with the additional branch, and then process PAR_2 is executed. This synchronization is meaningless from an external point of view, it is why we hide it in process PAR_1 


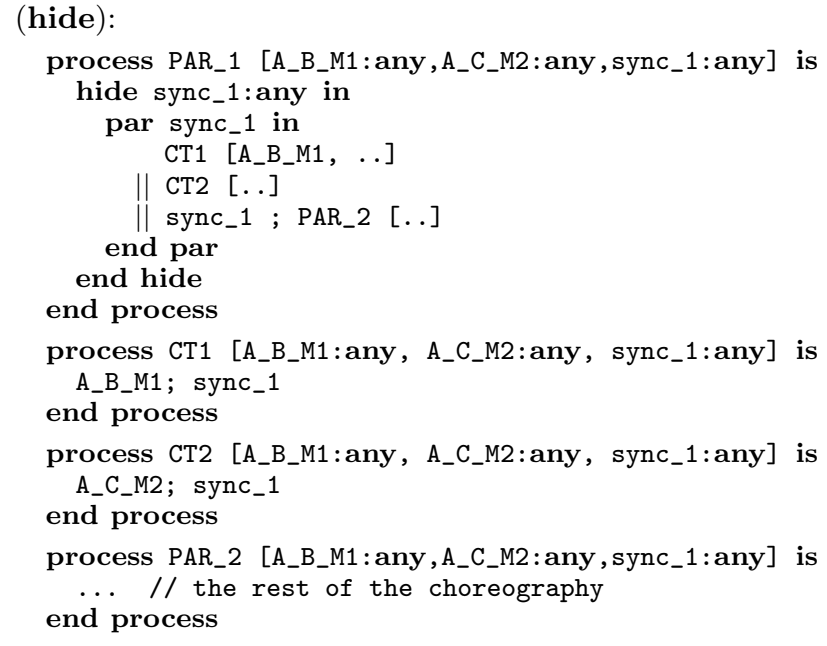

\section{REALIZABILITY}

From the LOTOS NT encoding of a BPMN choreography, we can generate the corresponding LTS model (Fig. 8) using the CADP state space exploration tools. This LTS was obtained by hiding "sync_" messages (which result in $\tau$ transitions), and by minimizing the resulting LTS using a weak trace reduction to remove $\tau$ transitions and determinize the LTS, and a strong reduction to suppress identical paths. In

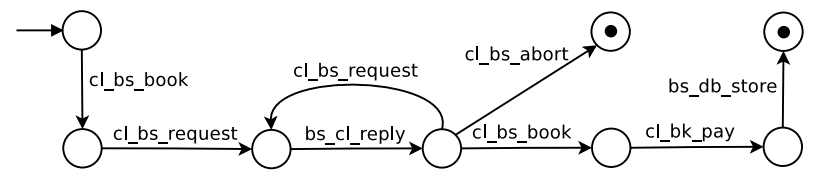

Figure 8: Booking System - LTS model

addition to these reduction techniques, all the CADP verification tools can be used to analyze this LTS. For instance, we can write and check temporal logic properties using the Evaluator model checker [23]. Concerning our running example, we can check that a client can make a booking or abort only if a request has been issued (safety property):

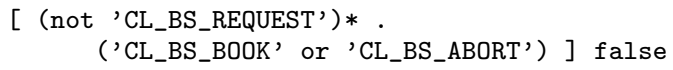

As for realizability, intuitively, a choreography is realizable if the set of interactions specified in BPMN and those executed by the interacting peers (obtained by projection from the choreography description) are the same. In this paper, we propose to check the realizability by comparing the choreography model with the model of the system composed of interacting peers using behavioural equivalences. If these two models are equivalent, it means that the peer generation exactly preserves the BPMN communication requirements. If they are not, it is because peers do not generate the same interactions as those specified in the choreography, therefore it is unrealizable. Here, we use strong equivalence [24] because LTS models for peers are minimized using the same reductions as for the choreography LTS.

Realizability checking is performed in several steps. First, we generate the choreography LTS from the LOTOS NT encoding as presented above. Second, we generate peers' behaviours. Third, we build the system composed of these interacting peers; some additional FIFO buffers are necessary if an asynchronous communication model is assumed. Finally, we check that the choreography LTS and the distributed version of the system are equivalent.

Synchronous communication. In order to generate peers declaratively, we extend our LOTOS NT encoding by generating a LOTOS NT process for each peer. We show below the LOTOS NT process generated for the booking system. We can see that each peer process calls the MAIN process encoding the choreography in LOTOS NT as presented in Section 3. Moreover, we hide all messages in which the peer at hand is not involved, e.g., we hide the payment (cl_bk_pay) between the client and the on-line bank in the peer corresponding to the booking system (peer_bs):

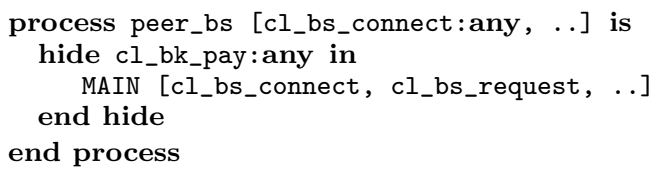

Finally, a LOTOS NT process is generated to specify the composition of peers and to make explicit the messages on which they synchronize. Going back to our running example, we show below how such a composition is obtained. First, peer bk is synchronized on cl_bk_pay with the rest of the system. Then, peer db synchronizes on bs_db_store with the two remaining peers, and so on:

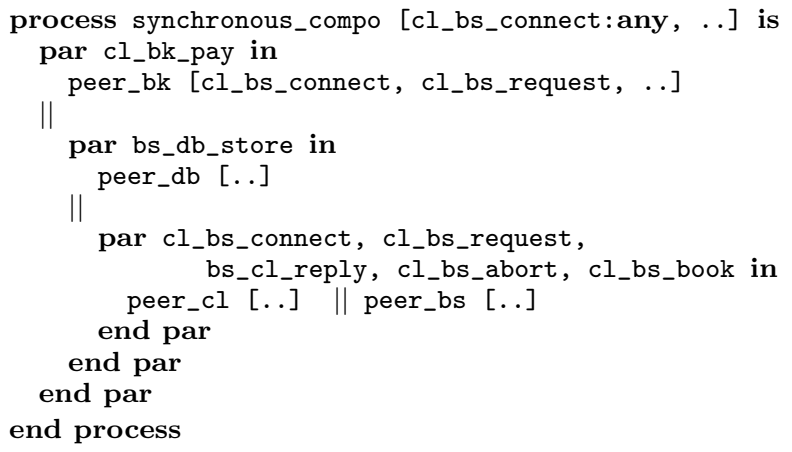

Asynchronous communication. Asynchronous communication is not directly supported by LOTOS NT. Therefore, we generate additional LOTOS NT code to implement bounded FIFO buffers and support asynchronous communication while preserving the message ordering (since realizability is sensitive to it). Buffers are described using the functional part of the LOTOS NT specification language, and handled through specific processes. Each peer process is associated with a buffer process from which it can consume messages received beforehand. A buffer process can also receive messages from other peers, and store them. Note that a local communication between a peer and its buffer has the suffix “_REC", whereas a communication between a peer (sender) and a buffer does not have a suffix. Finally, as for synchronous communication, we generate a process corresponding to the composition, which makes explicit the way peers and buffers interact.

This is demonstrated in Figure 9 for the database (db) peer. We also show below an excerpt of our running example showing how couples (peer,buffer) are generated for each peer. We see that the peer (apeer_db) and its buffer (buffer_db) are composed in parallel (par) and can interact on bs_db_store_REC, meaning that the peer can read this message from its buffer. The buffer has also the message 


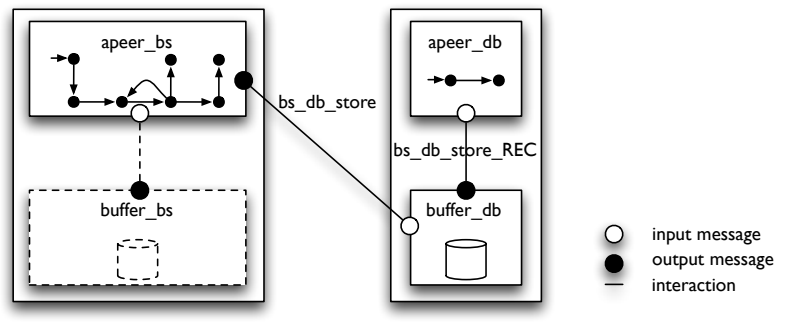

Figure 9: System architecture: communication between peers and buffers

bs_db_store in its alphabet corresponding to the interaction between the booking system and the database buffer on that message:

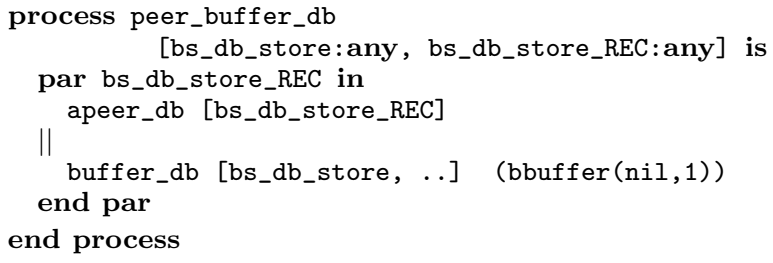

Many verification problems are undecidable when asynchronous communication is assumed [5]. In our framework, the user can either choose bounds for buffers ( 1 in the example above), or check for synchronizability [3]: a set of services is synchronizable if and only if the ordering of message exchanges remain the same when asynchronous communication is replaced with synchronous communication. Hence, if a system is synchronizable, the verification with CADP can be done on the synchronous version of the system and the results hold for the asynchronous case (without requiring any arbitrary bound).

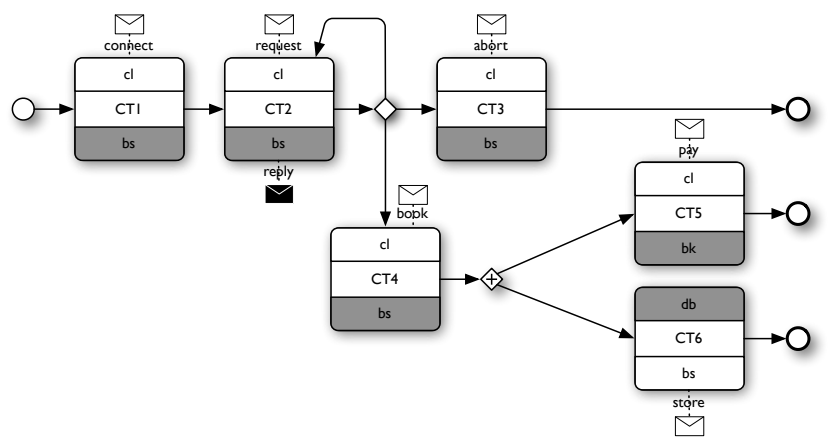

Figure 10: Booking System - version 2

Running example. Once we have generated all the required LOTOS NT processes, we use equivalence checking to compare on one hand the choreography LTS, and on the other the LTS generated for the distributed implementation of the system. As far as our running example is concerned, the equivalence check returns false for both communication models (synchronous and asynchronous), and indicates that the trace consisting of messages cl_bs_connect, cl_bs_request, bs_cl_reply, cl_bs_book appears in both systems, but bs_db_store is then present in the distributed system (it should not be), and not in the choreography LTS (see Fig. 8). The problem here is that messages cl_bk_pay and bs_db_store are executed independently from one another in the distributed system, whereas the choreography specification imposes an order between these messages. Since these two interactions are independent, the simplest solution is to make it explicit in the BPMN choreography by using a diverging parallel gateway as shown in Figure 10. In such a case, our realizability check returns positive results whatever communication model is chosen.

\section{TOOL SUPPORT AND EXPERIMENTS}

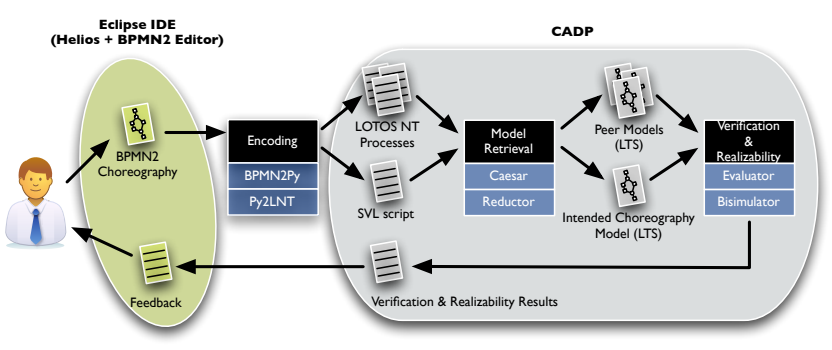

Figure 11: Tool support

Our approach is fully tool-supported as shown in Figure 11. In order to foster the usability of the approach, we have built it on top of the BPMN 2.0 Editor Eclipse plug-in ${ }^{1}$. Business process designers and service architects can use this plug-in to specify a BPMN choreography. A transformation from the XML representation of the choreography into an intermediary python format that we have developed is then performed using BPMN2Py, a .jar java runnable archive. BPMN2Py also calls Py2LNT, a python script that generates both the encoding of the choreography in LOTOS NT and a CADP SVL script that automates the remaining steps to be done for verification and realizability checking. The first step, i.e., the retrieval of LTS formal models for the peers, for their composition, and for the intended choreography behaviour, is achieved using the Caesar compiler and Reductor. The second step, i.e., verification, equivalence checking, and hence realizability, is achieved using Evaluator and Bisimulator. In case where realizability (or verification) does not yield, a counterexample is passed back to the designer in the form of a sequence of events $x_{-} y_{-} m$ ( $x$ sends message $m$ to $y$ ), enabling him/her to change the choreography. We have developed the BPMN2Py and Py2LNT tools, and we reuse the SVL script engine, and CADP state space generation and verification tools (model checker, equivalence checker, etc.).

Experiments. Table 1 shows experimental results on some examples of our database. Experiments have been carried out on a Xeon W3550 (3.07GHz, 12GB RAM) running Linux. For each experiment, the table gives the size of the BPMN choreography (in terms of number of peers, interactions, and gateways), the size of the LTS generated for the synchronous and asynchronous versions of the distributed system, the result of the synchronizability check, the realizability results for both communication models, and the overall execution time necessary to generate these LTSs and

\footnotetext{
${ }^{1}$ Eclipse update site: http://codehoop.com/bpmn2.
} 
compute these three checks. If the synchronizability property is not satisfied, we use 1-bounded buffers to check the choreography realizability. Example c0053 corresponds to the running example presented in this paper. It is worth observing that the number of peers involved in the composition as well as the number of parallel gateways used in the choreography specification increases the parallelism degree of the system and makes LTS sizes and computation times augment quickly (c0102).

\section{RELATED WORK}

Quite some work has already been dedicated to the realizability issue. The results presented in [9, 26] formalise wellformedness rules to enforce the specification to be realizable. More precisely, in [9], Carbone et al. identify three principles for global description under which they define a sound and complete end-point projection that is the generation of distributed processes from the choreography description. In [26], Qiu et al. propose a choreography language with new constructs (named dominated choice and loop) in order to implement unrealizable choreographies. During the projection of these new operators, communications are added in order to make peers respect the choreography specification. However, these solutions only focus on synchronous communication, and, similarly to what has been chosen for BPMN 2.0 choreographies [25], complicate the design by obliging the designer to make explicit extra-constraints in the choreography specification, e.g., by associating dominant roles to certain peers.

In [13], Decker and Weske propose a Petri Net-based formalism for specifying choreographies, and define realizability and local enforceability. They also propose algorithms to check these two properties. However, they consider only synchronous communication, and have not investigated mappings to higher-level interaction modeling languages (such as BPMN) yet.

Few works focused on the realizability problem assuming asynchronous communication. Fu et al. [15] proposed three sufficient conditions (lossless join, synchronous compatible, autonomous) that guarantee a realizable conversation protocol. More recently, Basu and Bultan proposed to check conformance using synchronizability [3]: A set of peers is synchronizable if systems produced on one hand with synchronous communication, and on the other with 1-bounded asynchronous communication, are equivalent. If a set of peers is synchronizable, one can check whether it is conformant to a choreography using existing finite state verification tools. Synchronizability complements the techniques proposed here, as presented in Section 4. In [6], Bravetti and Zavattaro tackle the choreography conformance issue from a theoretical point of view, and propose notions of contract refinement and choreography conformance for services that communicate through message queues.

Bultan and $\mathrm{Fu}$ [7] defined sufficient conditions to test realizability of choreographies specified with UML collaboration diagrams (CDs). In [27], Salaün and Bultan refine and extend this work with techniques to enforce realizability by adding additional synchronization messages among peers, and a tool-supported approach to automatically check the realizability of CDs for bounded asynchronous communication. The realizability problem for Message Sequence Charts (MSCs) has also been studied (e.g., [1, 28, 2]). [2] presents some decidability results on bounded MSC graphs, which are graphs obtained from MSCs using bounded buffers. These solutions are limited in the BPMN 2.0 context, because branching and cyclic behaviours are not well supported by CDs and MSCs (no choice in CDs, no cyclic behaviours in MSCs, and only self-loops in CDs). BPMN choreographies provide a more expressive notation than CDs or MSCs.

Decker and Weske present in [14] an extension of BPMN (iBPMN) in the direction of interaction modeling. They also propose a formal semantics for iBPMN in terms of interaction Petri nets. At the end of this paper, the authors mention realizability as a novel challenge, but do not give any solution for this issue. Lohmann and Wolf [20] show how realizability can be verified by using existing techniques for the controllability problem, which checks whether a service has compatible partner processes. They mention several models that can be used for modeling choreographies, such as iBPMN, but present their results on multi-peer automata called choreography automata. Their approach works for peers interacting via arbitrary bounded buffers, and only consider finite conversations. Here, we focused on the translation of high-level notations (BPMN) to a process algebra (LOTOS NT) for formal analysis purposes. This encoding, as well as the realizability check, are tricky issues, and even error-prone if manually done. This is the reason why some automated techniques and tools are absolutely essential.

\section{CONCLUDING REMARKS}

In this paper, we have focused on the part of the BPMN notation dedicated to the choreography design. We have proposed an encoding of a significative subset of BPMN choreographies into the LOTOS NT process algebra. This allows designers to validate their models using the CADP verification tools. They can also check whether their choreography model is realizable or not. If realizability is not ensured, a counter-example is returned which identifies a source of unrealizability. Our approach is fully automated and has been validated on many examples.

As far as perspectives are concerned, we would like to extend the subset of BPMN choreographies accepted by our approach with hierarchical structuring aspects (subchoreography). In this work, we chose a strong notion of realizability based on behavioural equivalence. Looser realizability notions have been investigated [18], and we plan to integrate them in our framework. We would also like to use recent compositional aggregation techniques [11] to reduce the intermediate state spaces size and improve the realizability checking computation times. We would like to propose a smart projection in order to enforce realizability. If a choreography is unrealizable, we would automatically generate peers extended with the minimum number of synchronization points to make them respect the choreography requirements. Finally, this work is a proof of concept on the automatic checking of realizability for the new BPMN 2.0 choreography notation. Within the context of a project on assisted electronic procedure fulfillment based on local (orchestration) and global (choreographic) contracts, we plan to experiment with our approach on a real-size case-study in the e-governance domain.

Acknowledgements. This work is supported by the Personal Information Management through Internet project (PIMI-ANR-2010-VERS-0014-03) of the French National Agency for Research. 


\begin{tabular}{|c|c|c|c|c|c|c|c|c|c|}
\hline \multirow{2}{*}{$\begin{array}{l}\text { BPMN } \\
\text { choreo. }\end{array}$} & \multicolumn{3}{|c|}{ Size } & \multirow{2}{*}{$\begin{array}{l}\text { Sync. compo. } \\
\text { LTS (st./tr.) }\end{array}$} & \multirow{2}{*}{$\begin{array}{l}\text { Async. compo. } \\
\text { LTS (st./tr.) }\end{array}$} & \multirow{2}{*}{$\begin{array}{c}\text { Synchronizability } \\
\text { property }[3]\end{array}$} & \multicolumn{3}{|c|}{ Realizability } \\
\hline & peers & interactions & gateways & & & & sync. & async. & time \\
\hline $\mathrm{c0007}$ & $\overline{2}$ & $\overline{66}$ & $\overline{66}$ & $699 / 151$ & $192 / 381$ & $\sqrt{ }$ & $\overline{\sqrt{ }}$ & $\overline{\sqrt{ }}$ & $\overline{19 \mathrm{~s}}$ \\
\hline $\mathrm{c} 0025$ & 3 & 4 & 2 & $55 / 112$ & $143 / 358$ & $\sqrt{ }$ & $\sqrt{ }$ & $\sqrt{ }$ & $24 \mathrm{~s}$ \\
\hline $\mathrm{c} 0053$ & 4 & 7 & 1 & $360 / 1,058$ & $1,058 / 3,450$ & $\sqrt{ }$ & $x$ & $x$ & $29 \mathrm{~s}$ \\
\hline $\mathrm{c} 0085$ & 5 & 10 & 3 & $6,697 / 27,818$ & $30,598 / 148,320$ & $x$ & $\sqrt{ }$ & $x$ & $2 \mathrm{~m} 40 \mathrm{~s}$ \\
\hline $\mathrm{c0102}$ & 6 & 9 & 7 & $29,056 / 178,360$ & $97,312 / 625,160$ & $x$ & $x$ & $x$ & $68 \mathrm{~m} 39 \mathrm{~s}$ \\
\hline
\end{tabular}

Table 1: Realizability results for some case studies

\section{REFERENCES}

[1] R. Alur, K. Etessami, and M. Yannakakis. Inference of Message Sequence Charts. IEEE Transactions on Software Engineering, 29(7):623-633, 2003.

[2] R. Alur, K. Etessami, and M. Yannakakis. Realizability and Verification of MSC Graphs. Theoretical Computer Science, 331(1):97-114, 2005.

[3] S. Basu and T. Bultan. Choreography Conformance via Synchronizability. In Proc. of $W W W^{\prime} 11$, pages 795-804. ACM Press, 2011.

[4] M. Bozkurt, M. Harman, and Y. Hassoun. Testing Web Services: A Survey. Technical report, King's College London, 2010.

[5] D. Brand and P. Zafiropulo. On Communicating Finite-State Machines. J. ACM, 30(2):323-342, 1983.

[6] M. Bravetti and G. Zavattaro. Contract Compliance and Choreography Conformance in the Presence of Message Queues. In Proc. of WS-FM'08, volume 5387 of $L N C S$, pages 37-54. Springer, 2009.

[7] T. Bultan and X. Fu. Specification of Realizable Service Conversations using Collaboration Diagrams. Service Oriented Computing and Applications, 2(1):27-39, 2008.

[8] N. Busi, R. Gorrieri, C. Guidi, R. Lucchi, and G. Zavattaro. Choreography and Orchestration Conformance for System Design. In Proc. of Coordination'06, volume 4038 of LNCS, pages 63-81. Springer, 2006.

[9] M. Carbone, K. Honda, and N. Yoshida. Structured Communication-Centred Programming for Web Services. In Proc. of ESOP'07, volume 4421 of LNCS, pages 2-17. Springer, 2007.

[10] D. Champelovier, X. Clerc, H. Garavel, Y. Guerte, V. Powazny, F. Lang, W. Serwe, and G. Smeding. Reference Manual of the LOTOS NT to LOTOS Translator (Version 5.4). INRIA/VASY, 149 pages, 2011.

[11] P. Crouzen and F. Lang. Smart Reduction. In Proc. of FASE'11, volume 6603 of $L N C S$, pages 111-126. Springer, 2011.

[12] G. Decker, O. Kopp, and A. Barros. An Introduction to Service Choreographies. Information Technology, 50(2):122-127, 2008.

[13] G. Decker and M. Weske. Local Enforceability in Interaction Petri Nets. In Proc. of BPM'0\%, volume 4714 of $L N C S$, pages 305-319. Springer, 2007.

[14] G. Decker and M. Weske. Interaction-centric Modeling of Process Choreographies. Information Systems, 36(2):292-312, 2011.

[15] X. Fu, T. Bultan, and J. Su. Conversation Protocols: A Formalism for Specification and Verification of
Reactive Electronic Services. Theoretical Computer Science, 328(1-2):19-37, 2004.

[16] H. Garavel, F. Lang, R. Mateescu, and W. Serwe. CADP 2010: A Toolbox for the Construction and Analysis of Distributed Processes. In Proc. of TACAS'11, volume 6605 of $L N C S$, pages $372-387$. Springer, 2011.

[17] ISO. LOTOS - A Formal Description Technique Based on the Temporal Ordering of Observational Behaviour. Technical Report 8807, ISO, 1989.

[18] R. Kazhamiakin and M. Pistore. Analysis of Realizability Conditions for Web Service Choreographies. In Proc. of FORTE'06, volume 4229 of $L N C S$, pages 61-76. Springer, 2006.

[19] J. Li, H. Zhu, and G. Pu. Conformance Validation between Choreography and Orchestration. In Proc. of TASE'07, pages 473-482. IEEE Computer Society, 2007.

[20] N. Lohmann and K. Wolf. Realizability Is Controllability. In Proc. of WS-FM'09, volume 6194 of LNCS, pages 110-127. Springer, 2010.

[21] A. Marconi and M. Pistore. Synthesis and Composition of Web Services. In Proc. of SFM'09, volume 5569 of $L N C S$, pages 89-157. Springer, 2009.

[22] R. Mateescu, P. Poizat, and G. Salaün. Adaptation of Service Protocols using Process Algebra and On-the-Fly Reduction Techniques. In Proc. of ICSOC'08, volume 5364 of LNCS, pages 84-99. Springer, 2008.

[23] R. Mateescu and M. Sighireanu. Efficient On-the-Fly Model-Checking for Regular Alternation-Free Mu-Calculus. Sci. Comput. Programming, 46(3):255-281, 2003.

[24] R. Milner. Communication and Concurrency. International Series in Computer Science. Prentice Hall, 1989.

[25] OMG. Business Process Model and Notation (BPMN) - Version 2.0. January 2011.

[26] Z. Qiu, X. Zhao, C. Cai, and H. Yang. Towards the Theoretical Foundation of Choreography. In Proc. of $W W W^{\prime} 07$, pages 973-982. ACM Press, 2007.

[27] G. Salaün and T. Bultan. Realizability of Choreographies using Process Algebra Encodings. In Proc. of IFM'09, volume 5423 of LNCS, pages 167-182. Springer, 2009.

[28] S. Uchitel, J. Kramer, and J. Magee. Incremental Elaboration of Scenario-based Specifications and Behavior Models using Implied Scenarios. ACM Transactions on Software Engineering and Methodology, 13(1):37-85, 2004. 